\title{
Bound for the P-Condition Number of Matrices With Positive Roots
}

\author{
Philip J. Davis, Emilie V. Haynsworth, and Marvin Marcus
}

(September 27, 1960)

Let a matrix $A$ have positive roots $0<\lambda_{1} \leq \lambda_{2} \leq \ldots . \leq \lambda_{n}$. Upper and lower bounds for the $P$-condition number of $A, P=\lambda_{n} / \lambda_{1}$, are given in terms of $\operatorname{det} A$ and one other symmetric function of the roots.

\section{Introduction}

Suppose $A=\left(a_{i j}\right)$ is a nonsingular $n \times n$ matrix with roots, $\lambda_{i}(i=1, \ldots, n)$, ordered so that

$$
0<\left|\lambda_{1}\right| \leq\left|\lambda_{2}\right| \leq \ldots \leq\left|\lambda_{n}\right|
$$

For a wide class of matrices $A$, the ratio $\left|\lambda_{n}\right| /\left|\lambda_{1}\right|$ gives a rough measure of the probable accuracy of the computation of the inverse of $A$, or the solution of the system of equations for which $A$ is the matrix of coefficients.

This measure was evaluated in some detail by von Neumann and Goldstine $[5]^{1}$ and has been called by $J$. Todd $[2,3,4]$ the $P$-condition number of the matrix, i.e.,

$$
P=\frac{\left|\lambda_{n}\right|}{\left|\lambda_{1}\right|}
$$

In general the accuracy of the results is in proportion to the reciprocal of $P$.

In this paper we shall show that, if the roots of $A$ are all positive with

$$
0<\lambda_{1} \leq \lambda_{2} \leq \ldots \leq \lambda_{n}
$$

and the determinant of $A$, $\operatorname{det} A$, is known, together with one other symmetric function of the roots, upper and lower bounds for $P$ can be given in terms of these two known quantities.

More precisely, if the characteristic polynomial for the matrix $A$ is

$p(x)=x^{n}-c_{1} x^{n-1}+c_{2} x^{n-2}$

$$
-\ldots+(-1)^{n} c_{n}, c_{i} \geq 0 \quad(i=1, \ldots, n)
$$

and we know $c_{k}$ and $c_{n}=(\operatorname{det} A)$, there exist positive upper and lower bounds for the ratio

$$
P=\frac{\lambda_{n}}{\lambda_{1}}
$$

in terms of $c_{k}$ and $c_{n}$.

To simplify the notation in the statement and proof of the theorem we use the following device.

\footnotetext{
1 Figures in brackets indicate the literature references at the end of this paper.
}

Let

$$
c_{k}=c_{k}\left(\lambda_{1}, \ldots, \lambda_{n}\right)=\left(\begin{array}{l}
n \\
k
\end{array}\right) s_{k}\left(\lambda_{1} \ldots, \lambda_{n}\right)=\left(\begin{array}{l}
n \\
k
\end{array}\right) s_{k} ;
$$

and divide each root of $p(x)$ by $\left(s_{k}\right)^{1 / k}$. (This is equivalent to dividing each element of the matrix by $\left(s_{k}\right)^{1 / k}$.) We will call the new matrix "normalized with respect to $k$ " or simply the normalized matrix. Since the condition number, $P$, is the ratio of two roots, $P$ will not be affected by such a transformation. Let $D_{k}$ be the determinant of the normalized matrix. Then

$$
D_{k}=\frac{s_{n}}{\left(s_{k}\right)^{n / k}}=\frac{\left(\begin{array}{l}
n \\
k
\end{array}\right)^{n / k} c_{n}}{c_{k}^{n / k}} .
$$

From Hardy-Littlewood-Pólya [1] the numbers $s_{k}$ as defined in (4) satisfy the inequalities

$$
s_{1} \geq s_{2}^{1 / 2} \geq s_{3}^{1 / 3} \geq \ldots \geq s_{n}^{1 / n}
$$

(where equality holds only if all the $\lambda_{i}$ are equal) hence, by $(5), D_{k} \leq 1$.

\section{Statement and Proof of Theorem}

Theorem: If we have a matrix $A=\left(a_{i j}\right)$ with characteristic polynomial (2) and if the constant term and the kth coefficient are known, the following bounds hold for $P$,

$\frac{1}{D_{k}^{1 /(n-1)}} \leq P \leq \frac{1+\sqrt{1-D_{k}^{n-1}}}{1-\sqrt{1-D_{k}^{n-1}}}, \quad(k=1, \ldots, n-1)$

where $D$ is defined by (4) and (5).

If $k=1$ (i.e., the trace of the matrix is given) we can improve the upper bound:

$$
P \leq \frac{1+\sqrt{1-D_{1}}}{1-\sqrt{1-D_{1}}} ; \quad D_{1}=\frac{n^{n} c_{n}}{c_{1}^{n}} .
$$

Proof: Part 1-Upper bound. We prove (8) first, as the method used for this can be applied in the proof of (7).

Since $P=\lambda_{n} / \lambda_{1}$, eq (8) is equivalent to

$$
D_{1} \leq \frac{4 \lambda_{1} \lambda_{n}}{\left(\lambda_{1}+\lambda_{n}\right)^{2}}
$$


so from (5), we must show that

$$
\frac{\left(\lambda_{1}+\lambda_{n}\right)^{2}}{4 \lambda_{1} \lambda_{n}} \leq\left(\frac{s_{1}}{n \sqrt{s_{n}}}\right)^{n} \text {. }
$$

But the right side of $(10)$ is $\left(A_{n} / G_{n}\right)^{n}$ where $A_{n}$ is the arithmetic mean, and $G_{n}$ the geometric mean, of $\lambda_{1}, \ldots, \lambda_{n}$.

Thus, we can prove (10) by induction if we prove the following:

LEMMA:

$$
\left(\frac{A_{n}}{G_{n}}\right)^{n} \leq\left(\frac{A_{n+1}}{G_{n+1}}\right)^{n+1}
$$

where $A_{n+1}$ and $G_{n+1}$ are the arithmetic and geometric means of $\lambda_{1}, \ldots, \lambda_{n}, \lambda_{n+1}$. (The $\lambda_{i}$ are not assumed to be ordered with respect to size for this lemma.)

Proof of Lemma: Simplifying (11) we see we must prove

$$
\lambda_{n+1} A_{n}^{n} \leq A_{n+1}^{n+1} .
$$

But (12) follows from the arithmetic-geometric mean inequality (cf., Hardy, Littlewood, Pólya [1]),

$$
a_{1} a_{2} \ldots a_{n+1} \leq\left(\frac{a_{1}+a_{2}+\ldots+a_{n+1}}{n+1}\right),^{n+1}
$$

if we let $a_{i}=A_{n},(i=1, \ldots, n)$ and $a_{n+1}=\lambda_{n+1}$.

Thus (11) holds and (10) follows immediately using finite induction on $n$.

To prove (7) we note that, by the inequalities (6),

$$
D_{k}^{n-1} \leq D_{n-1}^{n-1}=\frac{s_{n}^{n-1}}{s_{n-1}^{n}}
$$

so it suffices to show that

$$
\frac{\left(\lambda_{1}+\lambda_{n}\right)^{2}}{4 \lambda_{1} \lambda_{n}} \leq \frac{1}{D_{n-1}^{n-1}}=\left(\frac{s_{n-1}}{s_{n}}\right)^{n} \cdot s_{n} .
$$

Now let $\mu_{1}=\lambda_{n}^{-1}, \ldots, \mu_{n}=\lambda_{1}^{-1}$ and we note that $0<\mu_{1} \leq \ldots \leq \mu_{n}$ and (13) becomes

$$
\frac{\left(\mu_{1}+\mu_{n}\right)^{2}}{4 \mu_{1} \mu_{n}} \leq\left(\frac{s_{1}\left(\mu_{1} \ldots \mu_{n}\right)}{s_{n}^{1 / n}\left(\mu_{1} \ldots \mu_{n}\right)}\right)^{n} .
$$

But (14) is the same as (10) with the $\mu$ 's substituted for the $\lambda$ 's, hence (13) holds and the general upper bound (7) is true for all values of $k$.

Proof: Part 2-Lower Bound. From the inequalities (6),

$$
D_{k}=\frac{s_{n}}{s_{k}^{n / k}} \geq \frac{s_{n}}{s_{1}^{n}}
$$

where, by the definition (4)

Hence

$$
s_{1} \leq \lambda_{n}, s_{n} \geq \lambda_{1}^{n-1} \lambda_{n} .
$$

$$
\frac{1}{D_{k}} \leq \frac{s_{1}^{n}}{s_{n}} \leq \frac{\lambda_{n}^{n}}{\lambda_{1}^{n-1} \lambda_{n}}=\left(\frac{\lambda_{n}}{\lambda_{1}}\right)^{n-1}
$$

which proves the lower bound in (7).

\section{Some Remarks}

In reference [6] T. Kato gives an upper bound for the $P$-condition number which in our notation is

$$
P<\frac{4}{D_{1}} .
$$

This is larger than the bound given in (8).

We derive several consequences from (7), (8). Suppose that we have a matrix with positive roots, which is normalized so that $c_{k}=\left(\begin{array}{l}n \\ k\end{array}\right)$, i.e., $s_{k}=1$. Then $D_{k}=\operatorname{det} A$, and we can write

$$
\frac{1}{(\operatorname{det} A)^{1 /(n-1)}} \leq P \leq \frac{1+{\sqrt{1-(\operatorname{det} A)^{n-1}}}_{1-\sqrt{1-(\operatorname{det} A)^{n-1}}}}{1}
$$

This implies that

$$
\begin{aligned}
& \operatorname{det} A \rightarrow 0 \text { if and only if } P \rightarrow \infty, \\
& \operatorname{det} A \rightarrow 1 \text { if and only if } P \rightarrow 1 \text {. }
\end{aligned}
$$

Hence, the $\operatorname{det} A$ behaves essentially as the reciprocal of the $P$-condition number and may be said to constitute a reasonable condition number of its own for such matrices. This lends substance to the popular feeling that for a properly defined class of normalized matrices the smallness of its determinan $t$ is accompanied by difficulty in inversion.

A second observation relates to least square approximations. Express the general problem in the language of inner product spaces. We are required to solve $\left\|y-\sum_{i=1}^{n} a_{i} x_{i}\right\|=$ minimum, where $y$ is given and $x_{i}$ are independent elements. The normal equations have matrix $\left(\left(x_{i}, x_{j}\right)\right)$ where $(p, q)$ designates the inner product. We can assume $x_{1}$ normalized: $\left(x_{i}, x_{i}\right)=\left\|x_{i}\right\|^{2}=1$. The Gram matrix $\left(\left(x_{i}, x_{j}\right)\right)$ is positive definite symmetric and hence falls within the scope of our inequality with $k=1, c_{1}=n$. Hence if $G$ is the Gram determinant $\left|\left(x_{i}, x_{j}\right)\right|$ we have

$$
\frac{1}{G^{1 /(n-1)}} \leq P \leq \frac{1+\sqrt{1-G}}{1-\sqrt{1-G}} \text {. }
$$

The quantity $G$, which acts as a "measure" of linear independence of $x_{1}, \ldots, x_{n}$, therefore also serves as a condition number for the normal equations.

\section{References}

[1] G. H. Hardy, J. E. Littlewood, and G. Polya, Inequalities (Camb. Univ. Press, Cambridge, England 1952).

[2] J. Todd, The Condition of a Certain Matrix, Proc. Cambridge Phil. Soc. 46, pp. 116-118 (1949).

[3] J. Todd, The condition of certain matrices I, Quart J. Mech. Appl. Math. 2, pp. 469-472 (1949).

[4] J. Todd, The condition of certain matrices II, Arch. Math 5, pp. 249-257 (1954).

[5] J. von Neumann and H. Goldstine, Numerical inverting of matrices of high order, Bull. Am. Math. Soc. 53, pp. 1021-1099 (1947).

[6] T. Kato, Estimation of Iterated Matrices, with application to the von Neumann condition, Numerische Math. 2, pp. 22-29 (1960).

(Paper 65B1-42) 